\title{
Application of Artificial Intelligence in Automation of Supply Chain Management
}

\author{
Rupa Dash \\ University of Pittsburgh \\ Mark McMurtrey \\ University of Central Arkansas \\ Carl Rebman
University of San Diego \\ Upendra K. Kar \\ University of Pittsburgh
}

A well-functioning supply chain is a key to success for every business entity. Having an accurate projection on inventory offers a substantial competitive advantage. There are many internal factors like product introductions, distribution network expansion; and external factors such as weather, extreme seasonality, and changes in customer perception or media coverage that affects the performance of the supply chain. In recent years Artificial Intelligence (AI) has been proved to become an extension of our brain, expanding our cognitive abilities to levels that we never thought would be possible. Though many believe AI will replace humans, it is not true, rather it will help us to unleash our true strategic and creative potential. AI consists of a set of computational technologies developed to sense, learn, reason, and act appropriately. With the technological advancement in mobile computing, the capacity to store huge data on the internet, cloud-based machine learning and information processing algorithms etc. AI has been integrated into many sectors of business and been proved to reduce costs, increase revenue, and enhance asset utilization. AI is helping businesses to get almost $100 \%$ accurate projection and forecast the customer demand, optimizing their $R \& D$ and increase manufacturing with lower cost and higher quality, helping them in the promotion (identifying target customers, demography, defining the price, and designing the right message, etc.) and providing their customers a better experience. These four areas of value creation are extremely important for gaining competitive advantage. Supply-chain leaders use AI-powered technologies to a) make efficient designs to eliminate waste b) real-time monitoring and error-free production and c) facilitate lower process cycle times. These processes are crucial in bringing Innovation faster to the market. 


\section{INTRODUCTION}

Supply chain management (SCM) is one of the most challenging fields which emphasizes interactions among different sectors, primarily marketing, logistics, and production. Therefore, success in SCM lies in the overall success of any business. However, with the consistent changes in business practices like lean management and just-in-time philosophy both in production and logistics, globalization, adverse events i.e. frequent natural disaster, political instability, etc. SCM always need to develop an adequate solution to mitigate such challenges. In recent years technologies like Artificial Intelligence (AI) is been proved immensely valuable to SCM.

As the name suggests AI defined as the ability of a computer to independently solve problems that they have not been explicitly programmed to address. The field of AI came to existence in 1956, in a workshop organized by John McCarthy (McCarthy Et al., 2006). In successive years the pioneering work of McCarthy, Marvin Minsky, Nathaniel Rochester, and Claude Shannon, Arthur Samuel, Oliver Selfridge, Ray Solomonoff, Allen Newell, and Herbert Simon, etc. galvanized the field of "artificial intelligence" (Solomonoff, 1985). In his article "Computing Machinery and Intelligence" Alan Turing proposed the possibility of designing computers which can learn automatically (Turing, 1950). After "Shakey" a wheeled robot that was built at SRI, the field of mobile robotics gained International attention". However, in the late ' $90 \mathrm{~s}$ with the technological progress in designing computing power to store and process large dataset, the internet having the capacity to gather large amounts of data, and statistical techniques that, by design, can derive solutions from these data sets, allowed AI to emerge as one of the powerful technologies of the century (Kar et al, 2018). In the last two decades Technologies like Cognitive Computing, Computer Vision, Context-aware Computing, Natural Language Processing, Predictive Analytics, Machine Learning, Reinforcement Learning, Supervised Learning, Unsupervised Learning, and Deep Learning, etc. have enabled computer's "thoughts" by providing a conceptual framework for processing input and making decisions based on that data (Kar et al, 2018).

The modern machines enabled with AI platform are capable to gather information from its surroundings; using logic and probability choose to act with the highest likelihood of success. These machines are made to learn, and act intelligently based on the big-data sets and recognize objects or sounds with considerable precision (Mnih et al, 2015, Esteva et al., 2017). With the technological advancement in mobile computing, storage of huge data on the Internet and cloud-based machine learning and information processing algorithms, etc. applications and benefits of AI technologies are growing exponentially (Kar et al, 2018). Machines powered by AI performing many tasks — such as recognizing complex patterns, synthesizing information, drawing conclusions, and forecasting - that not long ago were assumed to require human cognition (Zhang et al.,1999, Bughin et al 2017). The best example would be Netflix and Amazon. Both companies use AI to personalize recommendations to millions of subscribers worldwide. From self-driving cars to implantable medical devices to electronic trading to a robot control of remote sensing are few other examples. Using deep learning algorithms, powered by advances in computation these machines have even exceeded human performance, particularly in visual tasks like playing Atari games (Perez et al., 2014), strategic board games like Go (Silver D et al., 2016) and object recognition (Esteva et al., 2017). AI, which enables machines to exhibit human-like cognition, therefore wherever a process uses digital data, AI can be applied to use that data more effectively to improve the functioning of most digital operations, products, and services (Hall DW et al., 2017). Applications of AI has helped businesses gain a competitive advantage in a) getting almost 100\% accurate projection and forecast the customer demand, b) optimizing their R\&D, therefore, increase in manufacturing with lower cost and higher quality c) helping them in the promotion (identifying target customers, demography, defining the price, and designing the right message, etc. d) providing their customers a better experience (has been explained in great detail in a later section). AI already in use in various business practices including medicine, law, finance, accounting, tax, audit, architecture, consulting, customer service, manufacturing, and transport, etc. (Hall DW et al., 2017). In this article, we have highlighted the recent trends and applications of AI in supply chain management, particularly in 
context to the retail and manufacturing industry. The examples given are the only representative in the respective areas.

\section{Application of AI in Value Creation}

Computer-based forecasting/demand planning is not new. It is based on a series of algorithms designed which takes various data sets like shipment data, product life cycle data, ordering pattern, manufacturing data, etc. over a period of time to forecast. In contrast, the AI enabled system knows the best possible combinations of algorithms and data sets to consider having an accurate prediction. More importantly, AI is helping businesses to a) get almost $100 \%$ accurate projection and forecast the customer demand, b) optimizing their R\&D, therefore, increase in manufacturing with lower cost and higher quality c) helping them in the promotion (identifying target customers, demography, defining the price, and designing the right message, etc. d) providing their customers a better experience. These four areas of value creation are extremely important for gaining competitive advantage.

\section{AI Helps to Forecast Demand and Optimization}

AI is been effectively used in projection and forecasting. Organizations are always keen to balance both supply and demand. Therefore, a better forecast is needed for its supply chain and manufacturing. As AI can process, analyze (automatically) and more importantly, predict data, it provides accurate and reliable forecasting demand, which allows businesses to optimize their sourcing in terms of purchases and orders processing therefore reducing costs related to transportation, warehousing and supply chain administration, etc. In addition, as it discerns trends and patterns which help to design better retailing and manufacturing strategies. For example; businesses use this tool in several ways, such as stock only the specific quantities (as accurate as each independent unit/product) of specific products they will sell and minimizing waste. Similarly; getting accurate sales trends they can order more soon-to-be-popular items. As these demand forecasts are so accurate they do not lose the sale because of product unavailability. National Grid in the United Kingdom uses the platform "DeepMind" developed by Google which predicts the best supply and demand variations accurately even considering variables like weather-related exogenous inputs (Yao 2017). Machine learning approaches not only incorporate historical sales data and the setup of the supply chains but also rely on near-real-time data regarding variables such as advertising campaigns, prices, and local weather forecasts (Bughin et al 2017). Otto a German online retailer manages to reduce $90 \%$ of their inventory using such application. The AI forecasts are so reliable that Otto building its inventory in anticipation of the orders, more interestingly totally relying on AI without any human intervention (Burgess, 2018). AI is also been used in R\&D departments, to quickly assess whether a prototype would be likely to succeed or fail in the market - and if so why. More importantly, it delivers more efficient designs by eliminating waste in the design process. By doing so AI has played an important role in smart manufacturing. (Kusiak A, 2018).

\section{AI Helps in the Production}

AI has played a significant role in production because a) better optimization of assets and processes, b) designing best teams i.e. people and robots, c) improvement in quality and reliability i.e. error-free, and d) prevention of downtime for maintenance. Automation process has taken a big stride because of AI technologies. Robotics one of the advanced branches of AI has taken a central role in the production (Bughin et al, 2017). Advances in technologies in object recognition and semantic segmentation has transformed the behavior of the robots, particularly in context to how they recognize the properties of the materials and objects they interact with. The new AI-enhanced, camera-equipped robots are trained to recognize empty shelf space. This leads to a dramatic speed advantage over conventional methods in picking objects (Bughin et al, 2017, Martin C et al. 2017). Deep learning has also been used to correctly identify an object and its position. This enables robots to handle objects without requiring the objects to be in fixed, predefined positions. Ocado, the UK supermarket, use one of the AI platforms in its retailer's warehouse, where robots steer thousands of product-filled bins over a maze of conveyor belts and deliver them to human packers just in time to fill 
shopping bags (Dale M., 2018). Similarly, other robots whisk the bags to delivery vans whose drivers are guided to customers' homes by the best route based on traffic conditions and weather (Bughin et al, 2017).

AI-enhanced logistics robots are also able to integrate disturbances in their movement routines via an unsupervised learning engine for dynamics. This capability leads to more precise makeovers and overall improved robustness of processes (Webster, $\mathrm{C}$ et al 2019). Collaborative robots can increase productivity by up to 20 percent (Bughin et al, 2017, Martin C et al. 2017). AI enabled semiconductor chip-production process is a good example of how AI helps in production. The cycle times from the first processing of the wafer to the final chip are typically several weeks to months and include various intermediate quality- testing processes. Testing costs and yield losses in semiconductor production can constitute up to 30 percent of the total production cost. Semiconductor manufacturers are using AI engines to identify root causes of yield losses that can be avoided by changing production processes. Enhanced applications are designed to monitor and adjust subprocesses in real time (Bughin et al, 2017, Martin C et al. 2017). AI techniques help not only determining the optimized product operating conditions or process conditions but also to significantly reduce defects in manufacturing. Similarly; in asset-heavy businesses, where complex systems running with minimal downtime, AI provides the perfect solution. Utility companies use AI for maintenance of their extensive electrical grids. Using data from sensors, drones, and other hardware, machine learning applications helps grid operators avoid decommissioning assets before their useful lives have ended, while simultaneously enabling them to perform more frequent remote inspections and maintenance to keep assets working well (Bughin et al, 2017). Using AI one European power distribution company reduced its cash costs as high as $30 \%$ over five years by replacing power transformers. AI is also enabling the "preventive maintenance" as well. Therefore, in a production unit where multiple machines are used, it will indicate the possible failure (Bughin et al, 2017, Martin C et al. 2017).

\section{AI helps in Promotion and Pricing}

Digital content has already become the norm and businesses employ multiple channels to reach their customers. About 25 percent of today's marketing budgets are devoted to digital channels, and almost 80 percent of marketing organizations make technology-oriented capital expenditures - typically hardware and software-according to a recent Gartner survey (Foo et al 2018, Sterne, 2018). AI-supported activities include digital advertising buys (programmatic buying), website operation and optimization, search engine optimization, A/B testing, outbound e-mail marketing, lead filtering and scoring, and many other marketing tasks (Sterne, 2018).

AI tools like Wordsmith, Articolo and Quill are already being used by the Associated Press and Forbes to create news, which leads to clicks on their websites (Seligman 2018). These tools use, templates, fill-in-the-blanks to enter data and keywords to create unique content which gives the readers the impression that a human has written it. AI is not only able to generate content; it can curate it. Content curation by AI not only connect the visitors with certain websites but also make recommendations based on their personal choice. Personalized email marketing campaigns based on preferences and user behaviors are well known (Sterne, 2018).

The Machine learning applications analyze millions of data about the behavior of consumer i.e. best frequency, what catches their attention the most and best times and days of the week to contact the user. A few of the AI-based applications like Boomtrain, Phrases, and Persado is already been shown their value. Phrases claims, the email it creates surpasses those of a human by over $95 \%$. The cognitive content of Persado demonstrated to exceed what a human could do 100\% of the time (Jaidka et al 2018, Gaggioli 2018). Similarly; Facebook, Amazon, and Google are well known for using AI enabled digital advertising (Deb et al 2018). The AI platform analyzes the information including interests, demographics, and other aspects to learn and predict the best audience for their brand. Adext (AI platform) can automate the handling and optimization of advertisements on various platforms including Google AdWords and Facebook. More importantly, it detects the most likely buyers and helps them to take the desired action or conversion. AI has revolutionized Internet searches and search engine optimization (SEO) (Deb et al 2018, Gaggioli 2018). AI devices like Amazon's Echo, Google's Home, Apple's Siri, and Microsoft's Cortana make it easy for their customers to perform searches by either saying a voice command or just pressing a button (Deb et al 2018). RankBrain developed by Google, can interpret the user's voice 
searches and, provides them the best results based on the user's language and context (Sutton et al 2018). Therefore, that famous long-tail keywords will be history. Smart marketers will use creative words to replace with more conversational keywords, which will increase their traffic and customers. An AI platform like Grid has transformed the webpage designing, the best part is it can customize the website for each customer and change the content of the website based on the preference of the user. Many brands already have chatbot powered by AI on their website. They serve clients $24 / 7$, more importantly, as they're fast they solve the problems of the customer faster than human. For example; Sephora uses an AI platform i.e. Visual Artist which identifies facial features and then uses augmented reality to analyze and suggest customized cosmetic products like lipsticks, eyeshadows, etc. (Kumar et al 2018).

Yield management programs were considered as the best system and been used for pricing airline seats, hotel rooms, and other perishables for years. However, AI has changed it dramatically. Now every business interested to know what price is the customer willing to pay? In a $24 \mathrm{X} 7$ connected world consumers continuously redefine value by comparing prices online, even when browsing in a brick-andmortar store. The right price at the right time increases customer satisfaction and leads to more sales and higher profit (Khorram 2019). Defining the optimal price for a product is complicated which is broadly depends on many factors including the day of the week, season, time of day, weather, channel and device, competitors' prices, etc. AI is a good tool to determine the price elasticity for every item and automatically adjust prices according to the chosen product strategy (Khorram 2019). In the retail industry, AI is been extensively used to optimize, update, and tailor it to each shopper in real time. AI program is been exploited which looked for clues about what the shopper will like based on previous purchases, age, home address, web browsing habits, and mounds of other data. This kind of insightsbased selling, including personalized promotions, optimized assortment, and tailored displays, increase sales substantially (Mathur 2019, Bughin et al, 2017). Aerospace companies are using AI technologies to prioritize sales targets and optimize the price of services. For years, they prioritized maintenance, repair, and overhaul (MRO) sales lead manually, a cumbersome, resource-heavy, and not always an efficient process. Using AI to improve the accuracy of forecasting MRO work and focusing on the firm's sales efforts on the most promising leads can have a significant effect on profitability (Kraus et al 2019, Bughin et al, 2017).

In recent years, artificial intelligence has enabled pricing solutions to track buying trends and determine more competitive product prices (Paolanti et al 2018). AI-driven pricing software has been included in various sectors including consumer goods, fashion, hospitality, and transportation (Meng et al 2018). In the future, businesses will progress from absolute i.e. static pricing to dynamic pricing which will offer customers different prices based on external factors and their individual buying habits. Dynamic pricing is based on aggregate available pricing data from various sources i.e. across the web, from competitors and prices are available in other regions. Dynamic pricing algorithms consider factors such as competitors' pricing, consumer behavior, location, time of day, and seasonality to determine how much shoppers are willing to pay for a product or service. Many machine learning programs have been designed to collect and analyzing data from a variety of sources like loyalty cards and postal codes, to predict what the customer is willing to pay and how responsive they might be to special offers. Most importantly, once the patterns are revealed, they can adjust and determine the best prices for new products that are appropriate for the customer (Kietzmann et al 2018).

Though dynamic pricing now in its infancy but will grow exponentially. Pace has developed a program that enables hotel management to design pricing that will match with supply and demand. This allows hotels to maximize their profits by offering the best price that customers are willing to pay based on various parameter including demographics, time of year, spending pattern, etc. It will help the hotels to forecast the demand (De Jesus 2019). Similarly; Shartsis has developed a "Perfect price" program which enables companies, such as car rental companies, to do dynamic pricing. Traditionally, car rental companies segment based on time of day, boosting morning prices to match business travelers who are assumed to be more willing to pay (De Jesus 2019). Perfect Price determines if a certain car shows higher demand in a specific area and at a specific time of day, resulting in surge pricing while not affecting other car classes. More importantly, the system needs minimal human oversight. In the future, using Google 
Tag Manager (GTM) and Perfect Price many businesses can adjust the product price. Incompetiror, developed by Intelligence Node is a retail product index that gives the user access to competitors' catalogs and pricing, allowing the user to use those prices as a benchmark for their own pricing structure (De Jesus 2019). Moreover, based on the application's product index, it allows businesses to view and compare their competitor's products to determine if theirs are overpriced, about equal, or underpriced. Similarly, using this information E-commerce business can know exactly which products shoppers want (De Jesus 2019).

They could access rich market data down to individual SKUs, product attributes, categories, and brands and gain visibility into product catalogs. There is another program "Wise Athena" which helps companies determine the best pricing for their products and trade promotion decisions. Wise Athena also claims to have the ability to automatically select a product's data attributes or specifications, and it computes for loss in sales volume, as well as the revenue or market share of a product when the same company launches a new product. It also computes for the potential change in the product's demand when the price for other product changes, as well as competition, leading price, and total sales (De Jesus 2019). Wise Athena reports that their system updates its machine learning models monthly to maintain or increase prediction accuracy. Navette PricePoint controls, manages, and measures pricing (De Jesus 2019). It consists of modules that group similar products, optimize local list prices from sales companies, dealers, and distributors, and takes into account incentives, discounts, and payment terms which help the businesses optimize prices. Business like Airbus, Tetra Pak, Olympus, Kia, and General Electric Healthcare are already using this program to design the price (De Jesus 2019). However, the role of AI in pricing is in its infancy and it will grow exponentially in the future.

\section{AI Helps in the Delivery}

Recently more focus has been given to "user experience" i.e. creating richer, more tailored, and more convenient for the user. Today's business is all about making every customer feel special and welcome, which is not an easy task. This used to be difficult and expensive and was often reserved for only the most lucrative clients. AI technologies like computer vision and machine learning has changed it completely. For example, a regular supermarket shopper puts a bunch of bananas in his cart, cameras or sensors could relay the information to an AI application that would have a good idea of what the shopper likes based on previous purchases. The app could then, via a video screen in the cart, suggest that bananas would be delicious with a chocolate fondue, which the purchase history suggests the shopper likes, and remind the shopper of where to find the right ingredients (Mortimer et al 2018, Bughin et al, 2017). Or a runner could download an app from an athletic shoe company, which would monitor her exercise regimen and recommend footwear tailored to her routine and running paths she may like. Amazon has built a retail outlet in Seattle that allows shoppers to take food off the shelves and walk directly out of the store without stopping at a checkout kiosk to pay (Metz 2018, Bughin et al, 2017). The store called Amazon Go, relies on computer vision to track shoppers after they swipe into the store and associate them with products taken from shelves. When shoppers leave, Amazon debits their accounts for the cost of the items in their bag and emails them a receipt (Metz 2018, Bughin et al, 2017). Delivery through drones is now a reality. Since Amazon successfully delivered a pilot delivery in rural England in 2016 there a surge in this area. Google partnered with Chipotle to deliver burritos at Virginia Tech, Dominos Pizza with Flirtey completed a commercial delivery of pizzas in New Zealand (Druehl et al 2018). UPS has partnered with drone company Zipline and governmental organizations in Africa to coordinate emergency medical supplies delivery (such as blood) in Rwanda (Druehl et al 2018). Amazon now routinely gathering data from drones during home delivery to target future purchases. From healthcare to education to transportation in every sector, AI is providing the ideal tools for operation management (Druehl et al 2018).

\section{AI in Smart Retailing}

AI enables the retail and manufacturing businesses in making smarter decisions, with more accurate and real-time forecasting, improving supply management, defining impactful thematic promotions, and 
optimizing assortment and pricing. AI also making operations more efficient, because of robotics and process optimization which enhances productivity and reduces manual labor costs. Use of interactive Robots in the warehouse and store are well known. The advancement in enhanced vision is enabled by more powerful computers, new algorithmic models, and large training data sets. Within the field of computer vision, object recognition and semantic segmentation - that is, the ability to categorize object type, such as distinguishing a tool from a component - have recently advanced significantly in their performance (Wen et al 2018, Bughin et al, 2017). They allow robots to behave appropriately for the context in which they operate, for example by recognizing the properties of the materials and objects they interact with. They are flexible and autonomous and capable of safely interacting with the real world and humans (Bughin et al, 2017). Companies like Swisslog, DHL, etc. are using these technologies efficiently (Wen et al 2018).

However, there are many hurdles yet to be crossed before we get the full potential of AI. The first and foremost is to gain the confidence of the stakeholders i.e. managers and employees, and those involved in the regulatory and policy-making boards. As discussed earlier robots are gradually being adapted to perform packaging and delivery. However; we still do not know how to address technical difficulties.

AI has enabled retailers to increase both the number of customers and the average amount they spend by creating personal and convenient shopping experiences. Retailers now know more about what their shoppers want - even before shoppers themselves (Deb et al 2018). AI forecasts from patterns and volumes of data i.e. previous transactions, weather forecasts, social media trends, shopping patterns, online viewing history, facial expression analysis, seasonal shopping patterns, etc (Fildes et al 2018, Burgess A 2018). The best examples are Amazon, Hulu, Netflix etc. Similarly; a European retailer improved its earnings before interest and taxes (EBIT) by 1 to 2 the percent by using a machine learning algorithm to anticipate fruit and vegetable sales. The company automatically orders more products based on this forecast to maximize turnover and minimize waste. A German company i.e. Otto cut surplus stock by 20 percent and reduced product returns by more than two million items a year, using deep learning to analyze billions of transactions and predict what customers will buy before they place an order (Burgess A 2018). AI technologies help retailers predict future store performance when expanding their physical footprints. Now retailers optimize their storage space and location using AI. Another important aspect of The retail industry is merchandising. AI helping in merchandising, with opportunities to improve assortment efficiency. Using geospatial and statistical modeling, they predict and minimize their stock. Amazon has embedded AI at the core of its operations. In the retailer's warehouse at Seattle, machine learning algorithms steer thousands of products over a maze of conveyor belts and deliver them to humans just in time to fill shopping bags. Other robots whisk bags to delivery vans whose drivers are guided by an AI application that picks the best route based on weather and traffic conditions (Fildes et al 2018, Burgess A 2018).

\section{AI in Smart Manufacturing}

Use of AI has transformed the manufacturing sector, from virtual assistants to advanced robotics, has enabled the manufacturing companies to produce more with fewer errors to adept demand. Using AI helped them in rapid growth as they can shorten development cycles, improve engineering efficiency, prevent faults, increase safety by automating risky activities, reduce inventory costs with better supply and demand planning, and increase revenue with better sales lead identification and price optimization, etc. (Patel et al 2018, Bughin et al, 2017). The new concept i.e. "Intelligent manufacturing" is a smart approach for production where machines are linked to humans i.e. both machine and humans are working side-by-side with minimal guidance. The best example of intelligent manufacturing is the manufacturing sector of Siemens ${ }^{2}$. The employee manages and controls the production of programmable logic circuits through a virtual factory that replicates the factory floor. Via barcodes, products communicate with the machines that make them, and the machines communicate among themselves to replenish parts and identify problems (Bughin et al, 2017). As high as $75 \%$ of the production process is fully automated, and 99.99988\% of the logic circuits are defect-free. Similarly, AI and 3D printing have revolutionized customization in manufacturing. Intel has developed Predictive analytics using machine learning a powerful tool to reduce the time required to solve design problems for semiconductor manufacturers (Burgess 2018). Motivo, an artificial intelligence startup, managed to compress semiconductor design 
processes from years to a few weeks, saving chip makers the cost of iterations and testing ${ }^{3}$. Using machine learning, aerospace manufacturing industries has developed productivity tools for engineering teams i.e. team travel norms, team composition, and supplier communication, etc. Machine learning has reduced its development costs by unleashing the speed, accuracy, and relevance of products. AI has allowed manufacturers to integrate production and client feedback in real time to refine the product design. With suppliers, AI-based tools provide better accountability throughout the supply chain, which helps aerospace manufacturers as well. For example, manufacturing a jet requires 1000 s of parts and procurement of these parts from around the world is a complex challenge. AI technologies like Virtual reality in manufacturing link thousands of different parts most importantly, it provides transparency on supplier machine availability, performance, and downtime, etc. It helps in balancing the supply chain and optimize inventories in real time (Kraus et al 2018).

Using AI manufacturers are optimizing the key performance indicators and review it in real time. For example; tailoring of a model using virtual programs help to better predict, identify, and prevent material and staffing bottlenecks and optimize energy consumption. More importantly, it alerts the engineers before problems arise and recommend solutions. AI enabled manufacturing not only efficiently assembly

line practices but also cut costs, reduce waste, and speed time to market (O'Reilly et al 2019). Using machine learning algorithms, collaborative robots, and self-driving vehicles have been proved to improve warehouse costs and reduce inventory levels as well. The best example is when General Electrics turned to Kaggle, the platform for predictive modeling and analytics competitions, and invited data scientists to design new routing and machine learning algorithms for flight planning that optimized fuel consumption by looking at variables such as weather patterns, wind, and airspace restraints. The winning routing algorithm showed a 12\% improvement in efficiency over actual flight data (Henriques et al 2018).

The key feature of AI enabled manufacturing is collaborative agility, which is the ability to adapt almost instantly to changes in demand and the evolution of regulation, input prices, technologies, and other parts of the industry landscape. Now smart Robots are working with humans collaboratively for mass production of products which is essential for customer-centric products. AI has helped manufacturing plants around the globe in supply chains, and value chains which are more interconnected and collaborative (Klumpp 2018). AI is also not far behind in agriculture. The concept of "e-plants in a box" is a reality which is great for small-scale, low-capital-expenditure, mobile plants that can produce a limited range of products at a competitive cost. Huxley combines machine learning, computer vision, and an augmented reality interface to essentially allow anyone to be a master farmer. More importantly, these e-plants can be transported to markets where demand is temporarily strong and in remote markets where production must be the local and low cost (Bughin et al, 2017).

\section{CONCLUSION}

The technological advancement in mobile computing, artificial neural networks, robotics, storage of huge data on the internet, cloud-based machine learning, and information processing algorithms, etc. has propelled the use of AI in various business sectors. Many businesses are using AI in major parts of their value chain as AI delivers significant competitive advantages. Most importantly, AI technologies have helped them eliminating many levels of manual activities including promotions, assortments, and supply chain. The e-commerce business using AI to predict the trends, optimize warehousing and logistics set prices, and personalize promotions etc. Some even go one step ahead like anticipating orders and shipping goods without even waiting for purchase confirmation. Similarly, Smart Manufacturing is now a reality. However, there are many changes are needed to fully get the benefit of AI, more importantly, the changes will compel many companies (retail and manufacture ring) to adopt new strategies i.e. plant designs, reshape their manufacturing footprints, and devise new supply chain models. Also, companies need to change the way they do business, as there will be a transition from human operators to AI enabled machines and robots. It is good to note that the trend in global industrial operation driven by AI is exponentially increasing, which suggests AI has either already or becoming a priority for many corporations around the world. 


\section{ENDNOTES}

1. Shaky the Robot. https://www.sri.com/work/timeline-innovation/timeline.php?timeline=computingdigital\#!\&innovation=shakey-the-robot.

2. Artificial intelligence in industry: intelligent production.

https://new.siemens.com/global/en/company/stories/industry/ai-in-industries.html

3. New specialized AI chips may revolutionize the \$1.6-plus trillion market cap semiconductor industry. What are the implications? https://latest.13d.com/specialized-ai-chips-revolutionize-semiconductor- industrytech-6b825d292282

\section{REFERENCES}

Al-Zahrani, A., \& Marghalani, A. (2018, April 25). How Artificial Intelligent Transform Business. Retrieved from https://ssrn.com/abstract=3226264 or http://dx.doi.org/10.2139/ssrn.3226264

Bughin, J., Hzan, E., Ramaswamy, S., Chui, M., et al. (2017, June). Artificial intelligence: The next digital frontier? McKinsey Global Institute.

Burgess, A. (2018). AI in Action. In: The Executive Guide to Artificial Intelligence. Palgrave Macmillan, Cham.

Burgess, J., Mitchell P., Highfield T. Automating the digital everyday: an introduction. November 14, 2017.

Dale M. (2018). Automating grocery shopping. Imaging and Machine Vision Europe, 85, 16+. Davenport, T.H. (2018). The AI Advantage: How to Put the Artificial Intelligence Revolution to Work.

De Jesus, A. (2019). AI for Pricing - Comparing 5 Current Applications. Retrieved from https://emerj.com/ai-sector-overviews/ai-for-pricing-comparing-5-current-applications/2019

Deb, S.K., Jain, R., \& Deb, V. (2018). Artificial Intelligence -Creating Automated Insights for Customer Relationship Management. 8th International Conference on Cloud Computing, Data Science \& Engineering (Confluence).

Druehl, C., Carrillo, J., \& Hsuan, J. (2018). Innovation and Supply Chain Management Technological Innovations: Impacts on Supply Chains. Springer.

Esteva, A., Kuprel, B., Novoa, R.A., Ko, J., Swetter, S.M., et al. (2017, February 2). Dermatologist-level classification of skin cancer with deep neural networks. Nature, 542(7639), 115-118.

Fildes, R.A., Ma, S., \& Kolassa, S. (2018, October 25) Retail forecasting: research and practice. Working Paper. Lancaster, 15, 10.

Foo, K.L., \& Virzi, A.M. (2018, July 19). Marketing Data and Analytics Survey 2018: Messy Data and Mismatched Resources Undermine Marketing Teams. Gartner research.

Gaggioli, A. (2018). Virtual Personal Assistants: An Emerging Trend in Artificial Intelligence. Cyberpsychology, Behavior, and Social Networking, 21(12).

Hall, D.W., \& Pesenti, J. (2017). Growing the Artificial Intelligence Industry in the UK. Henriques, R., \&

Feiteira I. (2018). Predictive Modelling: Flight Delays and Associated Factors, Hartsfield-Jackson Atlanta International Airport. Procedia Computer Science, 138, 638-645.

Jaidka, K., Goyal, T., \& Chhaya, N. (2018). Proceedings of the 10th ACM Conference on Web Science, 177-184.

Kar, U.K., \& Dash, R. (2018). The Future of Health and Healthcare in a World of Artificial Intelligence. Archives in Biomedical Engineering \& Biotechnology.

Khorram, M., Faria, P., Abrishambaf, O., \& Vale, Z. (2019). Demand Response Implementation in an Optimization Based SCADA Model Under Real-Time Pricing Schemes. In: Rodríguez S. et al. (eds) Distributed Computing and Artificial Intelligence, Special Sessions, 15th International Conference. DCAI 2018. Advances in Intelligent Systems and Computing, vol 801. Springer, Cham. 
Kietzmann, J., Paschen, J., \& Treen, E. (2018, September 1). Artificial Intelligence in Advertising: How Marketers Can Leverage Artificial Intelligence Along the Consumer Journey.10.2501/JAR-2018035.

Klumpp, M. (2018). Automation and artificial intelligence in business logistics systems: human reactions and collaboration requirements. International Journal of Logistics Research and Applications, 21(3).

Kraus, J., Lališ, A., Plos, V., Vittek, P., \& Stojić S. (2018). Utilizing Ontologies and Structural Conceptual Models for Safety Data Management in Aviation Maintenance, Repair and Overhaul Organizations. Transportation Research Procedia, 35, 35-43.

Kumar, K.S., Tamilselvan, S., \& Sha B. (2018, March). Artificial Intelligence Powered Banking Chatbot. International Journal of Engineering Science and Computing.

Kusiak, A. (2018). Smart manufacturing, Volume 56. International Journal of Production Research, 508517.

Martin, C., \& Leurent, H. (2017). Technology and Innovation for the Future of Production: Accelerating Value Creation. WEF.

Mathur, P. (2019). Key Technological Advancements in Retail. In: Machine Learning Applications Using Python. Apress, Berkeley, CA.

McCarthy, J., Minsky, M.L., Rochester, N., \& Shannon, C.E. (2006). A Proposal for the Dartmouth Summer Research Project on Artificial Intelligence. AI Magazine, 27(4).

Meng, F.J., Xu, J., Zhang, X., Yang, L., et. al. (2018). IEEE Symposium on Service-Oriented System Engineering (SOSE).

Metz, R. (2018). Amazon's cashier-less Seattle grocery store is opening to the public. MIT tech review.

Mnih, V., Kavukcuoglu, K., Silver, D., Rusu, A.A., et. al. (2015, February 26). Human level control through deep reinforcement learning. Nature, 518(7540), 529-33.

Mortimer, G., \& Milford, M. (2018, August 31) When AI meets your shopping experience it knows what you buy - and what you ought to buy. The Conversation.

O'Reilly, C., \& Binns, A.J.M. (2019). The Three Stages of Disruptive Innovation: Idea Generation, Incubation, and Scaling. California Management Review.

Paolanti, M., Liciotti, D., Pietrini, R. et al. (2018). J Intell Robot Syst, 91, 165. https://doi.org/10.1007/s10846-017-0674-7

Patel, P., Ali, M.I., \& Sheth, A. (2018). IEEE Intelligent Systems, From Raw Data to Smart Manufacturing: AI and Semantic Web of Things for Industry 4.0.

Perez, D., Samothrakis, S., \& Lucas, S. (2014). Knowledge-based fast evolutionary MCTS for general video game playing. IEEE Conference, 978-1-4799-3547-5.

Seligman, J. (2018). Artificial intelligence and machine learning and marketing management.

Silver, D., Huang, A., Maddison, C.J., Guez, A., et. al. (2016, January 28). Mastering the game of Go with deep neural networks and tree search. Nature, 529(7587), 484-9.

Solomonoff, R.J. (1985). The Time Scale of Artificial Intelligence: Reflections on Social Effects. Human Systems Management, 5(2), 149-153.

Sterne, J. (2018). From programming to statistics to machine learning for marketing. Applied Marketing Analytics, 3(4), 298-305(8).

Sutton, M. S., \& Griffiths, M. (2018). Using Date Specific Searches on Google Books to Disconfirm Prior Origination Knowledge Claims for Particular Terms, Words, and Names. Soc. Sci., 7(4), 66.

Turing, A. M. (1950). Computing Machinery and Intelligence. Mind, 59(236), 433-460.

Webster, C., \& Ivanov, S.H. (2019, February 17) Robotics, Artificial Intelligence, and the Evolving Nature of Work. In George, B., \& Paul, J. (Eds.). Business Transformation in Data Driven Societies, Palgrave-MacMillan (Forthcoming). Retrieved from SSRN: https://ssrn.com/abstract $=3336104$

Wen, J., He, L., Zhu, F., et. (2018). al Swarm Robotics Control and Communications: Imminent Challenges for Next Generation Smart Logistics. IEEE Communications Magazine, 56(7). 
Wodecki, A. (2018). Influence of Artificial Intelligence on Activities and Competitiveness of an Organization. Artificial Intelligence in Value Creation, 133-246.

Yao, W. (2017). Analysis on the Application of the Artificial Intelligence Neural Network on the New Energy Micro Grid. Proceedings of the 2017 4th International Conference on Machinery, Materials and Computer (MACMC).

Zhang, G., Hu, M.Y., Patuwo, B.E., \& Indro, D.C. (1999, July 1). Artificial neural networks in bankruptcy prediction: General framework and cross-validation analysis. European Journal of Operational Research, 116(1), 16-32. 